\title{
Three novel quinolinone alkaloids from the leaves of Melicope denhamii
}

Ratih Dewi Saputri, Rurini Retnowati, Unang Supratman, Tjitjik Srie Tjahjandarie \& Mulyadi Tanjung

To cite this article: Ratih Dewi Saputri, Rurini Retnowati, Unang Supratman, Tjitjik Srie Tjahjandarie \& Mulyadi Tanjung (2021): Three novel quinolinone alkaloids from the leaves of Melicope denhamii, Natural Product Research, DOI: 10.1080/14786419.2021.1960524

To link to this article: https://doi.org/10.1080/14786419.2021.1960524

View supplementary material $\widetilde{ }$

曲 Published online: 09 Sep 2021.

Submit your article to this journal $₫$

Q View related articles $₫$

View Crossmark data $\asymp$ 


\title{
Three novel quinolinone alkaloids from the leaves of Melicope denhamii
}

\author{
Ratih Dewi Saputri ${ }^{a}$, Rurini Retnowati ${ }^{\mathrm{b}}$, Unang Supratman ${ }^{\mathrm{c}}$, \\ Tjitjik Srie Tjahjandarie $^{d}$ and Mulyadi Tanjung ${ }^{d}$
}

${ }^{a}$ Department of Chemistry, Faculty of Mathematics and Natural Sciences, Universitas Negeri Surabaya, Surabaya, Indonesia; ${ }^{b}$ Department of Chemistry, Faculty of Mathematics and Natural Sciences, Universitas Brawijaya, Malang, Indonesia; 'Department of Chemistry, Faculty of Mathematics and Natural Sciences, Universitas Padjadjaran, Jatinangor, Indonesia; ${ }^{\mathrm{d}}$ Natural Products Chemistry Research Group, Organic Chemistry Division, Department of Chemistry, Faculty of Science and Technology, Universitas Airlangga, Surabaya, Indonesia

\section{ABSTRACT}

Three previously unreported quinolinone alkaloids: melicodenines $\mathrm{J}-\mathrm{L}$ (1-3) and six known compounds (4-9), were isolated from the leaves of Melicope denhamii (Seem) T.G. Hartley. The structures of three quinolinone alkaloids were identified based on HRESIMS and NMR spectra. Compounds 1-9 were assayed in three cancer cells (MCF-7, HeLa, and P-388). Compounds $\mathbf{1}$ and $\mathbf{5}$ showed high cytotoxic activity against HeLa cells with $\mathrm{IC}_{50}$ values of 1.8 and $0.8 \mu \mathrm{M}$, respectively.

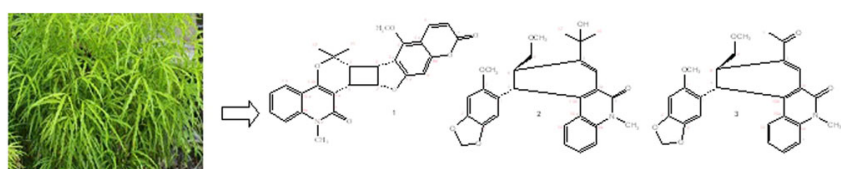

\section{ARTICLE HISTORY}

Received 12 May 2021

Accepted 18 July 2021

\section{KEYWORDS}

Melicope denhamii; melicodenines $\mathrm{J}-\mathrm{L}$; quinolinone adduct; cytotoxic

\section{Introduction}

M. denhamii (Seem) T.G. Hartley (Rutaceae) is one small tree indigenous to Java Islands, Indonesia. The Melicope genus produces alkaloids (Chen et al. 2003; Nakashima et al. 2011), flavonoids (Saputri et al. 2018), and phenylpropanoids (Nakashima et al. 2012), with terpenyl side chain in the aromatic ring. Many alkaloids from Melicope show biological activities as cytotoxic agents (Chen et al. 2003; Nakashima et al. 2012), and antimalaria (Rasamison et al. 2016). Recently studies on Melicope resulted in the hybrid compound by [2+2] cycloaddition and a Diels-Alder adduct from incorporated phenylpropanoid-phenylpropanoid, alkaloid-alkaloid, alkaloid-benzopyran, and alkaloid-phenylpropanoid derivatives (Nakashima et al. 2011, 2012; George et al. 2016; Saputri et al. 2021). Three new compounds, melicodenine J (1) is a [2+2] cycloaddition, melicodenines $\mathrm{K}(\mathbf{2})$, and $\mathrm{L}(\mathbf{3})$ are Diels-Alder adduct

CONTACT Mulyadi Tanjung mulyadi-t@fst.unair.ac.id

(-) Supplemental data for this article can be accessed online at https://dx.doi.org/10.1080/14786419.2021.1960524.

(C) 2021 Informa UK Limited, trading as Taylor \& Francis Group 
derivatives were isolated from $M$. denhamii leaves. The cytotoxic activities of their isolates (1-9) against MCF-7, HeLa, and P-388 cancer cell lines were reported in this study.

\section{Result and discussion}

Melicodenine J (1) was isolated as a yellow amorphous solid and showed a positive ion peak $[\mathrm{M}+\mathrm{H}]^{+}$at $\mathrm{m} / \mathrm{z}$ 458.1613, consistent with the molecular composition $\mathrm{C}_{27} \mathrm{H}_{23} \mathrm{NO}_{6}$. The UV exhibited maximum absorption $\left(\lambda_{\max } 219,259,292,320\right.$, and $334 \mathrm{~nm}$ ), indicating a typical quinolinone alkaloid-coumarin (Nakashima et al. 2012). The IR measurement showed absorption bands for conjugated carbonyl at $1627 \mathrm{~cm}^{-1}$, aromatic ring at $1595 \mathrm{~cm}^{-1}$, and ether at $1128 \mathrm{~cm}^{-1}$. The ${ }^{1} \mathrm{H}$ NMR spectrum of 1 showed four protons $\left[\delta_{\mathrm{H}} 5.41\left(1 \mathrm{H}, d d, J=6.7,2.6 \mathrm{~Hz}, \mathrm{H}-2^{\prime}\right), \delta_{\mathrm{H}} 4.75(1 \mathrm{H}, t, J=6.7 \mathrm{~Hz}, \mathrm{H}-\right.$ $\left.\left.3^{\prime}\right), \delta_{\mathrm{H}} 4.08(1 \mathrm{H}, t, J=9.5 \mathrm{~Hz}, \mathrm{H}-4), \delta_{\mathrm{H}} 3.10(1 \mathrm{H}, d d, J=9.5,6.7 \mathrm{~Hz}, \mathrm{H}-3)\right]$ were characteristics of a 1,2,3,4-tetrasubstituted cyclobutane ring. A signal at $\delta_{\mathrm{H}} 5.41$ indicates an oxymethine attached to the cyclobutane ring (Holla et al. 2012; Nakashima et al. 2012). Four signals of a 1,2-disubstituted benzene $\left[\delta_{\mathrm{H}} 7.90(1 \mathrm{H}, d d, J=8.0,1.2 \mathrm{~Hz}, \mathrm{H}-10), \delta_{\mathrm{H}}\right.$ $7.43(1 \mathrm{H}, d t, J=8.5,1.2 \mathrm{~Hz}, \mathrm{H}-8), \delta_{\mathrm{H}} 7.15(1 \mathrm{H}, t, J=8.0 \mathrm{~Hz}, \mathrm{H}-9), \delta_{\mathrm{H}} 7.08(1 \mathrm{H}, d$, $J=8.5 \mathrm{~Hz}, \mathrm{H}-7)]$, two methyls $\left[\delta_{\mathrm{H}} 1.73(3 \mathrm{H}, \mathrm{s}, \mathrm{H}-11), \delta_{\mathrm{H}} 1.20(3 \mathrm{H}, \mathrm{s}, \mathrm{H}-12)\right]$ along with a $\mathrm{N}$-methyl signal at $\delta_{\mathrm{H}} 3.38$ suggested that the partial structure of $\mathbf{1}$ as a $\mathrm{N}$-methylflindersin moiety (Kamperdick et al. 1999). A signal of aromatic at $\delta_{\mathrm{H}} 5.94\left(1 \mathrm{H}, \mathrm{s}, \mathrm{H}-9^{\prime}\right)$, two signals of cis vinylic $\left[\delta_{\mathrm{H}} 7.85\left(1 \mathrm{H}, d, J=9.6 \mathrm{~Hz}, \mathrm{H}-5^{\prime}\right), \delta_{\mathrm{H}} 5.95(1 \mathrm{H}, d, J=9.6 \mathrm{~Hz}, \mathrm{H}-\right.$ $\left.6^{\prime}\right)$ ], and a methoxyl at $\delta_{\mathrm{H}} 4.25\left(3 \mathrm{H}, \mathrm{s}, 4^{\prime}-\mathrm{OCH}_{3}\right)$ recommended that the other partial structure of 1 as a bergapten moiety (Saputri et al. 2021). Based on the ${ }^{1} \mathrm{H}$ NMR data suggested that the structure of $\mathbf{1}$ is a [2+2] cycloaddition product between N-methylflindersin with bergapten (Nakashima et al. 2012). The ${ }^{13} \mathrm{C}$ NMR and HMQC spectra of 1 exhibited the signals of 27 carbons were completely separated, including four methyls $\left[\delta_{\mathrm{C}} 25.4,25.5,29.0,58.4\right], 11$ methines $\left[\delta_{\mathrm{C}} 35.5,43.2,45.0,85.1,90.5,109.5,113.5\right.$, $121.6,123.1,130.7,139.5]$, five quaternary carbons $\left[\delta_{C} 104.4,105.2,107.3,116.2\right.$, $138.5]$, one oxycarbon $\left[\delta_{\mathrm{C}} 75.5\right]$, two carbonyls $\left[\delta_{\mathrm{C}} 161.8,162.2\right]$, and four oxyaryls $\left[\delta_{\mathrm{C}}\right.$ $152.6,156.5,156.6,168.6]$. The HMBC spectrum, an N-methyl signal at $\delta_{\mathrm{H}} 3.38$, showed a correlation with a carbonyl $\left[\delta_{\mathrm{C}} 162.2(\mathrm{C}-5)\right]$ and a quaternary carbon $\left[\delta_{\mathrm{C}} 138.5\right.$ (C6a)]. An aromatic signal at $\delta_{\mathrm{H}} 7.90(\mathrm{H}-10)$ correlated to $\mathrm{C}-6 \mathrm{a}$ and a methine carbon $\left[\delta_{\mathrm{C}}\right.$ $130.7(\mathrm{C}-8)]$. Two methyl signals at $\delta_{\mathrm{H}} 1.73(\mathrm{H}-11)$ and $\delta_{\mathrm{H}} 1.20(\mathrm{H}-12)$ correlated to an oxycarbon $\left[\delta_{\mathrm{C}} 75.5(\mathrm{C}-2)\right]$, and a methine carbon $\left[\delta_{\mathrm{C}} 45.0(\mathrm{C}-3)\right]$ proved that a part of the structure of $\mathrm{N}$-methylflindersin. A signal of vinylic at $\delta_{\mathrm{H}} 7.85\left(\mathrm{H}-5^{\prime}\right)$ showed correlation with a lactone carbonyl $\left[\delta_{\mathrm{C}} 161.8\left(\mathrm{C}-7^{\prime}\right)\right]$, two oxyaryls $\left[\left(\delta_{\mathrm{C}} 152.6\left(\mathrm{C}-4^{\prime}\right)\right.\right.$, and $\left(\delta_{\mathrm{C}}\right.$ 156.5, C-8á)]. A methoxyl at $\delta_{\mathrm{H}} 4.25\left(4^{\prime}-\mathrm{OCH}_{3}\right)$ correlated to $\mathrm{C}-4^{\prime}$ verified the location of the methoxyl group at $\mathrm{C}-4^{\prime}$. One proton of aromatic at $\delta_{\mathrm{H}} 5.94\left(\mathrm{H}-9^{\prime}\right)$ showed correlation with two oxyaryls [( $\delta_{\mathrm{C}} 168.6, \mathrm{C}-9$ á), C-8á) $]$, two quaternary carbons $\left[\left(\delta_{\mathrm{C}} 104.4, \mathrm{C}-\right.\right.$ $3 a ́)$, and $\left.\left(\delta_{\mathrm{C}} 107.3, \mathrm{C}-4 a \hat{)}\right)\right]$ and carbonyl carbon $\left(\delta_{\mathrm{C}} 161.8, \mathrm{C}-7^{\prime}\right)$ reinforced the other partial structure of 1 as a bergapten (Saputri et al. 2021). An oxymethine proton at $\delta_{\mathrm{H}}$ $5.41\left(\mathrm{H}-2^{\prime}\right)$ correlated to a methine carbon $\delta_{\mathrm{C}} 35.5(\mathrm{C}-4)$. A signal at $\delta_{\mathrm{H}} 4.75\left(\mathrm{H}-3^{\prime}\right)$ correlated to C-3á, C-4á, C-4', and C-9a' (a part of bergapten), C-3, and C-4 (a part of Nmethylflindersin). A methine signal of a cyclobutane ring at $\delta_{\mathrm{H}} 3.10(\mathrm{H}-3)$ correlated to 
C-4, C-3á, and a methine, $\delta_{\mathrm{C}} 85.1\left(\mathrm{C}-2^{\prime}\right)$. A methine signal of a cyclobutane ring at $\delta_{\mathrm{H}}$ $4.08(\mathrm{H}-4)$ correlated to $\mathrm{C}-4 \mathrm{a}$ and $\mathrm{C}-2^{\prime}$. In the NOESY spectrum, an oxymethine $\left(\mathrm{H}-2^{\prime}\right)$ correlated to $\mathrm{H}-3$ and $\mathrm{H}-3^{\prime}$, and a methine proton $(\mathrm{H}-3)$ correlated to $\mathrm{H}-4$ and $\mathrm{H}-3^{\prime}$ revealed the signal that a 1,2,3,4-tetrasubstituted cyclobutane ring is a cis orientation. Consequently, the structure of melicodenine $J$ is shown in Figure 1.

Melicodenine $\mathrm{K}(2)$ was isolated as colorless oil in which showed an ion peak $[\mathrm{M}+\mathrm{H}]^{+}$at $\mathrm{m} / \mathrm{z} 464.2080$ corresponding for a molecular composition $\mathrm{C}_{27} \mathrm{H}_{30} \mathrm{NO}_{6}$ by the combination of HRESIMS spectra and NMR data. The IR spectrum showed bands of conjugated carbonyl $\left(1639 \mathrm{~cm}^{-1}\right)$, aromatic $\left(1502\right.$ and $\left.1485 \mathrm{~cm}^{-1}\right)$, and ether $\left(1112 \mathrm{~cm}^{-1}\right)$ groups. The ${ }^{1} \mathrm{H}$ NMR spectrum of 2, showing four aromatic signals $\left[\delta_{\mathrm{H}} 7.52(1 \mathrm{H}, d d, J=8.6,1.2 \mathrm{~Hz}, \mathrm{H}-10), \delta_{\mathrm{H}} 7.46\right.$ $\left.(1 \mathrm{H}, t, J=7.7 \mathrm{~Hz}, \mathrm{H}-8), \delta_{\mathrm{H}} 7.35(1 \mathrm{H}, d, J=8.6 \mathrm{~Hz}, \mathrm{H}-7), \delta_{\mathrm{H}} 7.15(1 \mathrm{H}, t, J=7.7 \mathrm{~Hz}, \mathrm{H}-9)\right]$, an Nmethyl signal at $\delta_{\mathrm{H}} 3.80$, a vinylic at $\delta_{\mathrm{H}} 6.97(1 \mathrm{H}, \mathrm{s}, \mathrm{H}-4)$, and two methyls $\left[\delta_{\mathrm{H}} 1.49(3 \mathrm{H}, \mathrm{s}, \mathrm{H}-11)\right.$, $\left.\delta_{\mathrm{H}} 0.88(3 \mathrm{H}, \mathrm{s}, \mathrm{H}-12)\right]$ indicating for a 3-isoprenyl-1-methyl 2-quinolinone moiety (Chen et al. 2003). The ${ }^{1} \mathrm{H}$ NMR spectrum of 2 also exhibited two protons of aromatic $\left[\delta_{\mathrm{H}} 6.57\left(1 \mathrm{H}, \mathrm{s}, \mathrm{H}-3^{\prime}\right)\right.$, $\left.\delta_{\mathrm{H}} 6.13\left(1 \mathrm{H}, \mathrm{s}, \mathrm{H}-6^{\prime}\right)\right]$, two methines $\left[\delta_{\mathrm{H}} 4.82\left(1 \mathrm{H}, \mathrm{s}, \mathrm{H}-7^{\prime}\right), \delta_{\mathrm{H}} 3.19\left(1 \mathrm{H}, d d, J=9.8,6.0 \mathrm{~Hz}, \mathrm{H}-8^{\prime}\right)\right]$, splitting two signals of a methylene $\left[\delta_{\mathrm{H}} 3.51\left(1 \mathrm{H}, d d, J=8.3,6.0 \mathrm{~Hz}, \mathrm{H}-9\right.\right.$ á, $\delta_{\mathrm{H}} 3.38(1 \mathrm{H}, t$, $\left.\left.J=9.8 \mathrm{~Hz}, \mathrm{H}-9 \mathrm{~b}^{\prime}\right)\right]$, two methoxyls $\left[\delta_{\mathrm{H}} 3.95\left(3 \mathrm{H}, \mathrm{s}, 2^{\prime}-\mathrm{OCH}_{3}\right), \delta_{\mathrm{H}} 3.30\left(3 \mathrm{H}, \mathrm{s}, 9^{\prime}-\mathrm{OCH}_{3}\right)\right]$, and splitting two signals of a methylenedioxy $\left[\delta_{\mathrm{H}} 5.79(1 \mathrm{H}, d, J=1.2 \mathrm{~Hz}), \delta_{\mathrm{H}} 5.75(1 \mathrm{H}, d, J=1.2 \mathrm{~Hz})\right]$ characteristics for a melicodin A moiety (Nakashima et al. 2012). Compound 2 indicated that 27 carbon signals were utterly separated in the ${ }^{13} \mathrm{C}$ NMR spectra, including five methyl carbons, two methylene carbons, nine methine carbons, one carbonyl carbon, and ten quaternary carbons. From the NMR $\left({ }^{1} \mathrm{H},{ }^{13} \mathrm{C}\right)$ NMR spectrum exhibited that the structure of $\mathbf{2}$ is a Diels-Alder adduct moiety and was confirmed by HMBC spectrum (Nakashima et al., 2012; George et al. 2016). The HMBC correlation, an N-methyl at $\delta_{\mathrm{H}} 3.80$, and an aromatic at $\delta_{\mathrm{H}} 7.46$ $(\mathrm{H}-8)$ very similar to 1. A vinylic signal at $\delta_{\mathrm{H}} 6.97(\mathrm{H}-4)$ correlated to $\delta_{\mathrm{C}} 71.6(\mathrm{C}-2), \delta_{\mathrm{C}} 139.7(\mathrm{C}-$ $3), \delta_{\mathrm{C}} 39.1\left(\mathrm{C}-8^{\prime}\right)$, and $\delta_{\mathrm{C}} 160.6(\mathrm{C}-5)$. Two methyls at $\delta_{\mathrm{H}} 0.88(\mathrm{H}-12)$ and $\delta_{\mathrm{H}} 1.49(\mathrm{H}-11)$ correlated to $\mathrm{C}-2$, indicating the 3-isoprenyl 1-methyl 2-quinolinone moiety. Two signals of aromatic at $\delta_{\mathrm{H}} 6.57\left(\mathrm{H}-3^{\prime}\right)$, and $\delta_{\mathrm{H}} 6.13\left(\mathrm{H}-6^{\prime}\right)$ correlated to $\delta_{\mathrm{C}} 150.8\left(\mathrm{C}-2^{\prime}\right), \delta_{\mathrm{C}} 147.0\left(\mathrm{C}-4^{\prime}\right)$, and $\delta_{\mathrm{C}}$ $140.7\left(\mathrm{C}-5^{\prime}\right)$. A methylenedioxy signal $\left[\delta_{\mathrm{H}} 5.79\right.$ and $\left.\delta_{\mathrm{H}} 5.75\right]$ correlated to $\mathrm{C}-4^{\prime}, \mathrm{C}-5^{\prime}$ indicated fused at $\mathrm{C}-4^{\prime}$ and $\mathrm{C}-5^{\prime}$, a methoxyl at $\delta_{\mathrm{H}} 3.95\left(2^{\prime}-\mathrm{OCH}_{3}\right)$ correlated to $\mathrm{C}-2^{\prime}$. A methoxyl signal at $\delta_{\mathrm{H}} 3.30\left(9^{\prime}-\mathrm{OCH}_{3}\right)$ correlated to $\delta_{\mathrm{C}} 76.2\left(\mathrm{C}-9^{\prime}\right)$. Two signals of an aromatic, a methylenedioxy, two methoxyls are the signal of a melicodin A moiety. A methine at $\delta_{\mathrm{H}} 4.82\left(\mathrm{H}-7^{\prime}\right)$ correlated to $\delta_{\mathrm{C}} 149.3(\mathrm{C}-10 \mathrm{~b}), \delta_{\mathrm{C}} 124.9(\mathrm{C}-4 \mathrm{a}), \delta_{\mathrm{C}} 119.2\left(\mathrm{C}-1^{\prime}\right), \delta_{\mathrm{C}} 108.5\left(\mathrm{C}-6^{\prime}\right), \mathrm{C}-8^{\prime}$, and C-9'. A methine signal at $\delta_{\mathrm{H}} 3.19\left(\mathrm{H}-8^{\prime}\right)$ correlated to $\mathrm{C}-3, \delta_{\mathrm{C}} 115.6(\mathrm{C}-4), \mathrm{C}-10 \mathrm{~b}, \mathrm{C}-1^{\prime}, \delta_{\mathrm{C}} 34.7\left(\mathrm{C}-7^{\prime}\right)$, and $\mathrm{C}-9^{\prime}$. The correlation of three methines $\left[\left(\delta_{\mathrm{H}} 4.82\left(\mathrm{H}^{\prime} 7^{\prime}\right), \delta_{\mathrm{H}} 3.19\left(\mathrm{H}-8^{\prime}\right)\right.\right.$, and $\left.\delta_{\mathrm{H}} 6.97(\mathrm{H}-4)\right]$, indicating the structure of $\mathbf{2}$ are Diels-Alder adduct. The NOE spectrum, the proton signal at $\mathrm{H}-3^{\prime}$ correlated with $\mathrm{H}-8^{\prime}$ and $2^{\prime}-\mathrm{OCH}_{3}$ exhibited that the proton signal at $\mathrm{H}-7^{\prime}$ and $\mathrm{H}-8^{\prime}$ revealed trans orientation, and the relative configuration of $\mathbf{2}$ was similar to melicodenine $\mathrm{H}$ (Nakashima et al. 2012). The structure of melicodenine $K(\mathbf{2})$ is shown in the Figure 1.

Melicodenine $L(3)$ was obtained as a yellowish oil, showing an ion peak $[\mathrm{M}+\mathrm{H}]^{+}$ at $\mathrm{m} / \mathrm{z}$ 448.1752, conforms for a molecular composition $\mathrm{C}_{26} \mathrm{H}_{25} \mathrm{NO}_{6}$ through HRESIMS spectra. The UV $\left(\lambda_{\max } 226,246,259,265,309 \mathrm{~nm}\right), \operatorname{IR}(1636,1600,1552$, and 1119), and NMR $\left({ }^{1} \mathrm{H}\right.$ and $\left.{ }^{13} \mathrm{C}\right)$ of $\mathbf{3}$ had very identical with 2 . The significant difference in the $1 \mathrm{D}$ and 2D NMR, compound 3 showed an acetyl group at $\delta_{\mathrm{H}} 2.45(\mathrm{H}-1), \delta_{\mathrm{C}} 25.8(\mathrm{C}-1)$, and $\delta_{\mathrm{C}} 198.2(\mathrm{C}-2)$. The HMBC and HMQC experiments assigned the acetyl group at C-1 

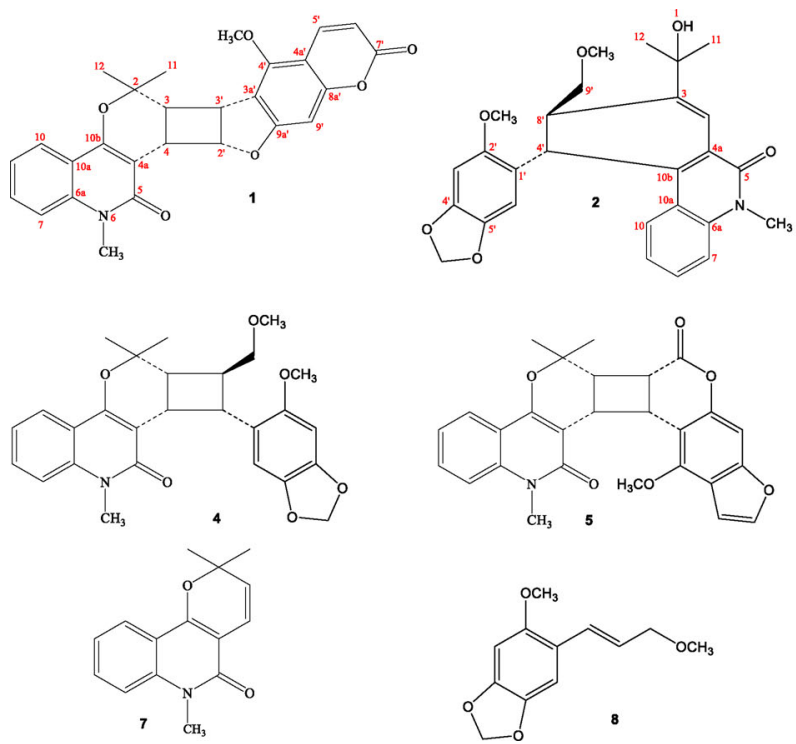
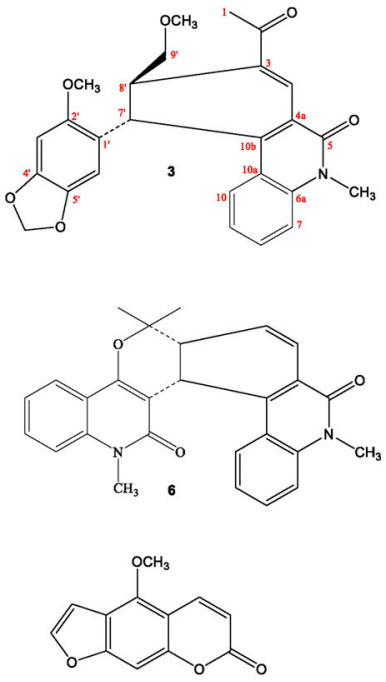

9

Figure 1. Structures of compounds 1-9 from M. denhamii.

Table 1. Cytotoxic activities of the isolated compounds from M. denhamii.

\begin{tabular}{lccc}
\hline & \multicolumn{1}{c}{$\mu \mathrm{M}$} & P-388 \\
\cline { 2 - 4 } Compounds & MCF-7 & HeLa & $>100$ \\
\hline Melicodenine J (1) & $>100$ & $1.8 \pm 0.02$ & $29.1 \pm 1.10$ \\
Melicodenine K (2) & $>100$ & $62.9 \pm 1.45$ & $>100$ \\
Melicodenine L (3) & $>100$ & $>100.9 \pm 1.13$ & $11.9 \pm 0.87$ \\
Melicodenine E (4) & $>100$ & $0.8 \pm 0.15$ & $38.3 \pm 1.42$ \\
Melicodenine F (5) & $>100$ & $>100$ & $13.9 \pm 0.65$ \\
Melicobisquinolinone B (6) & $>100$ & $>100$ & $87.2 \pm 0.30$ \\
N-methylflindersin (7) & $>100$ & $>100$ & $>100$ \\
Melicodin A (8) & $>15.0 \pm 0.15$ & $0.9 \pm 0.04$ & $>100$ \\
Bergapten (9) & $>0.8 \pm 0.02$ & - & - \\
Doxorubicin & - & & $1.3 \pm 0.07$ \\
Artonin E & & &
\end{tabular}

and C-2. The methyl proton at $\delta_{\mathrm{H}} 2.45$ correlated with a carbonyl [ $\delta_{\mathrm{C}} 198.2$ (C-2)] in the $\mathrm{HMBC}$ spectrum. A signal of $\alpha, \beta$-unsaturated ketone at $\delta_{\mathrm{H}} 8.09(\mathrm{H}-4)$ correlated to $\mathrm{C}-2, \delta_{\mathrm{C}} 146.2(\mathrm{C}-10 \mathrm{~b}), \delta_{\mathrm{C}} 135.9(\mathrm{C}-3), \delta_{\mathrm{C}} 160.2(\mathrm{C}-5)$, and $\delta_{\mathrm{C}} 37.3\left(\mathrm{C}-8^{\prime}\right)$. The NOE spectrum of 3 , showing the relative configurations very similar to melicodenine $\mathrm{K}$. Therefore, the structure of melicodenine $L(3)$ in Figure 1. In conclusion, melicodenine $L(\mathbf{3})$ is demethylation and is followed by an oxidation reaction of $\mathbf{2}$.

Six known compounds, melicodenine $E(4), F(5)$, melicobisquinolinone $B(6), N$ methylflindersin (7), melicodin A (8), and bergapten (9), elucidating by comparing their NMR spectra based on the chemical shift that reported (Johns et al. 1968; Kamperdick et al., 1999; Nakashima et al. 2011, 2012).

In vitro evaluation against MCF-7, HeLa, and P-388 for their activities in accord with the MTT method (Table 1) uses artonin E and doxorubicin as a positive control. The cells without active compound as a negative control (Tanjung et al. 2018; Tjahjandarie et al. 2021). Melicodenines J (1) and F (5) exhibited very high activity against HeLa. A 
type Diels-Alder adduct (2, 3, 6), a type monomer, was inactive (7-9). However, compounds 1-9 were inactive on MCF-7 and P-388 cancer cells (Table 1). A type $[2+2]$ cycloaddition adduct $(\mathbf{1}, \mathbf{4}, \mathbf{5})$ plays a key role for the cytotoxic effect. The effect of the bond angle of the cyclobutane ring more than active the cyclohexene ring inhibiting the growth of HeLa cells.

\section{Experimental}

\subsection{Plant material}

The collecting of the fresh leaves of $M$. denhamii came from Tanah Merah, Bangkalan, Madura Island, East Java, Indonesia, in Feb 2016. The plant was identified by a senior botanist (I. Rachman) from the Bogoriense Herbarium, Indonesia. A specimen (MD 20171207) was deposited as a reference.

\subsection{Extraction and isolation}

The dried leaves of $M$. denhamii $(3.1 \mathrm{~kg}$ ), extracted with $\mathrm{MeOH}$ two times $(10 \mathrm{~L}$, each for three days) at room temperature, and the $\mathrm{MeOH}$ extract $(100 \mathrm{~g})$ treated with $5 \%$ aqueous $\mathrm{H}_{2} \mathrm{SO}_{4}(\mathrm{pH} \mathrm{3-4)}$ and then partitioned with $n$-hexane $(18 \mathrm{~g})$, and EtOAc $(15 \mathrm{~g})$, respectively. The acid layer was treated with $\mathrm{NH}_{4} \mathrm{OH}(\mathrm{pH}$ 8-9) and extracted with EtOAc to give alkaloid extract $(1 \mathrm{~g})$. The alkaloid extract $(4.8 \mathrm{~g})$, fractionated by radial planar chromatography on silica gel, using a gradient of $n$-hexane-EtOAc (from 9:1 to 1:1 $\mathrm{v} / \mathrm{v})$ to afford two significant fractions, $A(188 \mathrm{mg})$ and $B(450 \mathrm{mg})$. Purification of fraction $A$ by radial planar chromatography, eluted with $n$-hexane-acetone (from 9:1 to 4:1 v/v), gave compound 7 (88 mg). Fraction B $(450 \mathrm{mg})$, further separated by CC chromatography on Sephadex LH-20, eluted with methanol, gave two subfractions, $B_{1}$ $(253 \mathrm{mg})$ and $B_{2}(75 \mathrm{mg})$. Subfraction $B_{1}$ separated with radial planar chromatography, eluted with $n$-hexane-EtOAc (from 9:1 to 7:3 v/v), gave compounds 1 (9.8 $\mathrm{mg}$ ), 6 $(25 \mathrm{mg})$, and $2(6.2 \mathrm{mg})$. Similarly, subfraction $B_{2}$ separated by the same method, eluted with $n$-hexane- $\mathrm{CHCl}_{3}$ (from 7:3 to $3: 7 \mathrm{v} / \mathrm{v}$ ), afforded compound 3 (4.8 mg), compound $4(5 \mathrm{mg})$, and compound $5(6 \mathrm{mg})$. The EtOAc extract $(14 \mathrm{~g})$, fractionated by VLC on silica gel, using a gradient of $n$-hexane-EtOAc (from 9:1 to 3:7 v/v), gave four significant fractions, C-F. Fraction $C(800 \mathrm{mg})$ further separated by radial planar chromatography on silica gel, eluted with $n$-hexane- $\mathrm{CHCl}_{3}(4: 1$ to $1: 1 \mathrm{v} / \mathrm{v})$, afforded compound 8 ( $27 \mathrm{mg})$. Fraction E $(205 \mathrm{mg})$ by the same method, eluted with $n$-hexanediisopropyl ether (7:3 to $3: 7 \mathrm{v} / \mathrm{v})$, afforded compound 9 (16 mg).

\subsection{Spectral data}

Melicodenine J (1): yellow solid, m.p. $224-225^{\circ} \mathrm{C},[\alpha]_{\mathrm{D}}^{20}=+6^{\circ}$ (c $\left.0.0005, \mathrm{MeOH}\right)$ : UV $(\mathrm{MeOH}) \lambda_{\max }(\log \varepsilon) 219$ (4.48), 259 (3.83), 292 (3.83), 320 (4.06), and $334 \mathrm{~nm}$ (4.03). IR $(\mathrm{KBr}) \mathrm{V}_{\max }\left(\mathrm{cm}^{-1}\right) 1627,1595,1461$, and 1128. ${ }^{1} \mathrm{H}-\mathrm{NMR}\left(\mathrm{CDCl}_{3}, 4100 \mathrm{MHz}\right), \delta_{\mathrm{H}} \mathrm{ppm}$ : $7.90(1 \mathrm{H}, d d, J=8.0,1.2 \mathrm{~Hz}, \mathrm{H}-10), 7.85\left(1 \mathrm{H}, d, J=9.6 \mathrm{~Hz}, \mathrm{H}-5^{\prime}\right), 7.43(1 \mathrm{H}, d t, J=8.5$, $1.2 \mathrm{~Hz}, \mathrm{H}-8), 7.15(1 \mathrm{H}, t, J=8.0 \mathrm{~Hz}, \mathrm{H}-9), 7.08(1 \mathrm{H}, d, J=8.5 \mathrm{~Hz}, \mathrm{H}-7), 5.95(1 \mathrm{H}, d$, $\left.J=9.6 \mathrm{~Hz}, \mathrm{H}-6^{\prime}\right), 5.94\left(1 \mathrm{H}, \mathrm{s}, \mathrm{H}-9^{\prime}\right), 5.41\left(1 \mathrm{H}, d d, J=6.7,2.6 \mathrm{~Hz}, \mathrm{H}-2^{\prime}\right), 4.75(1 \mathrm{H}, t$, 
$\left.J=6.7 \mathrm{~Hz}, \mathrm{H}-3^{\prime}\right), 4.25\left(3 \mathrm{H}, s^{\prime} 4^{\prime}-\mathrm{OCH}_{3}\right), 4.08(1 \mathrm{H}, t, J=9.5 \mathrm{~Hz}, \mathrm{H}-4), 3.38\left(3 \mathrm{H}, \mathrm{s}, \mathrm{N}-\mathrm{CH}_{3}\right)$, $3.10(1 \mathrm{H}, d d, J=9.5,6.7 \mathrm{~Hz}, \mathrm{H}-3), 1.73(3 \mathrm{H}, s, \mathrm{H}-11), 1.20(3 \mathrm{H}, s, \mathrm{H}-12) .{ }^{13} \mathrm{C}-\mathrm{NMR}\left(\mathrm{CDCl}_{3}\right.$, $100 \mathrm{MHz}$ ), $\delta_{\mathrm{C}}$ ppm: 168.6 (C-9á), 162.2 (C-5), 161.8 (C-7'), 156.6 (C-10b), 156.5 (C-8á), 152.6 (C-4'), 139.5 (C-5'), 138.5 (C-6a), 130.7 (C-8), 123.1 (C-10), 121.6 (C-9), 116.2 (C10a), 113.5 (C-7), 109.5 (C-6'), 107.3 (C-4á), 105.2 (C-4a), 104.4 (C-3á), 90.5 (C-9'), 85.1 $\left(\mathrm{C}-2^{\prime}\right), 75.5(\mathrm{C}-2), 45.0(\mathrm{C}-3), 43.2\left(\mathrm{C}-3^{\prime}\right), 35.5(\mathrm{C}-4), 29.0\left(6-\mathrm{NCH}_{3}\right), 25.5(\mathrm{C}-11), 25.4(\mathrm{C}-$ 12). HRESIMS $m / z 458.1613[\mathrm{M}+\mathrm{H}]^{+}$calculated for $\mathrm{C}_{27} \mathrm{H}_{23} \mathrm{NO}_{6} \mathrm{~m} / \mathrm{z} 458.1604$.

Melicodenine $\mathrm{K}(2)$ : colorless oil, $[\alpha]_{\mathrm{D}}^{20}=+8^{\circ}$ (c $\left.0.0005, \mathrm{MeOH}\right): \mathrm{UV}(\mathrm{MeOH}) \lambda_{\max }$ ( $\log$ \&) 229 (3.99), 259 (3.60), 308 (3.51), 325 (3.46), 359 (3.56) and $377 \mathrm{~nm}$ (4.41). IR $(\mathrm{KBr}) v_{\max }\left(\mathrm{cm}^{-1}\right) 1639,1502,1485$, and 1112. ${ }^{1} \mathrm{H}-\mathrm{NMR}\left(\mathrm{CDCl}_{3}, 400 \mathrm{MHz}\right), \delta_{\mathrm{H}} \mathrm{ppm}: 7.52$ $(1 \mathrm{H}, d d, J=8.6,1.2, \mathrm{H}-10), 7.46(1 \mathrm{H}, t, J=7.7 \mathrm{~Hz}, \mathrm{H}-8), 7.35(1 \mathrm{H}, d, J=8.6 \mathrm{~Hz}, \mathrm{H}-7), 7,15$ $(1 \mathrm{H}, t, J=7.7 \mathrm{~Hz}, \mathrm{H}-9), 6.97(1 \mathrm{H}, s, \mathrm{H}-4), 6.57\left(1 \mathrm{H}, s, \mathrm{H}-3^{\prime}\right), 6.13\left(1 \mathrm{H}, s, \mathrm{H}-66^{\prime}\right), 5.79$ and $5.75\left(2 \mathrm{H}, d, J=1.2 \mathrm{~Hz}, 4^{\prime}-\mathrm{O}-\mathrm{CH}_{2}-\mathrm{O}-5^{\prime}\right), 4.82\left(1 \mathrm{H}, \mathrm{s}, \mathrm{H}-7^{\prime}\right), 3.95\left(3 \mathrm{H}, \mathrm{s}, 2^{\prime}-\mathrm{OCH}_{3}\right), 3.80(3 \mathrm{H}$, $\left.s, \mathrm{~N}-\mathrm{CH}_{3}\right), 3.51\left(1 \mathrm{H}, d d, J=8.3,6.0 \mathrm{~Hz}, \mathrm{H}-9^{\prime} \mathrm{a}\right), 3.38\left(1 \mathrm{H}, t, J=9.8 \mathrm{~Hz}, \mathrm{H}-9{ }^{\prime} \mathrm{b}\right), 3.30(3 \mathrm{H}, s$, $\left.9^{\prime}-\mathrm{OCH}_{3}\right), 3.19\left(1 \mathrm{H}, d d, J=9.8,6.0 \mathrm{~Hz}, \mathrm{H}-8^{\prime}\right), 1.49(3 \mathrm{H}, \mathrm{s}, \mathrm{H}-11), 0.88(3 \mathrm{H}, \mathrm{s}, \mathrm{H}-12) .{ }^{13} \mathrm{C}-$ NMR (CDCl $\left.{ }_{3}, 100 \mathrm{MHz}\right), \delta_{\mathrm{C}} \mathrm{ppm}: 160.6(\mathrm{C}-5), 150.8\left(\mathrm{C}-2^{\prime}\right), 149.3(\mathrm{C}-10 \mathrm{~b}), 147.0\left(\mathrm{C}-4^{\prime}\right)$, 140.7 (C-5'), 139.7 (C-3), 139.3 (C-6a), 129.8 (C-8), 124.9 (C-4a/C-10), 122.5 (C-9), 120.2 (C-10a), $119.2\left(\mathrm{C}-1^{\prime}\right), 115.6(\mathrm{C}-4), 114.5(\mathrm{C}-7), 108.5\left(\mathrm{C}-6^{\prime}\right), 101.1\left(4^{\prime}-\mathrm{O}-\mathrm{CH}_{2}-\mathrm{O}-5^{\prime}\right), 94.4$ (C$\left.3^{\prime}\right), 76.2\left(\mathrm{C}-9^{\prime}\right), 71.6(\mathrm{C}-2), 59.0\left(9^{\prime}-\mathrm{OCH}_{3}\right), 56.6\left(2^{\prime}-\mathrm{OCH}_{3}\right), 39.1\left(\mathrm{C}-8^{\prime}\right), 34.7\left(\mathrm{C}-7^{\prime}\right), 30.0(\mathrm{C}-$ 11), $29.9\left(6-\mathrm{NCH}_{3}\right), 29.2(\mathrm{C}-12), \mathrm{HRESIMS} \mathrm{m} / \mathrm{z} 464.2080[\mathrm{M}+\mathrm{H}]^{+}$calculated for $\mathrm{C}_{27} \mathrm{H}_{30} \mathrm{NO}_{6} \mathrm{~m} / \mathrm{z} 464.2073$.

Melicodenine K (3): yellowish oil, $[\alpha]_{\mathrm{D}}^{20}=-8^{\circ}$ (c 0.0005, MeOH): UV (MeOH) $\lambda_{\max }$ $(\log \varepsilon) 226$ (4.38), 246 (4.20), 259 (4.07), 265 (3.65), and $309 \mathrm{~nm}$ (3.96). IR (KBr) $v_{\max }$ $\left(\mathrm{cm}^{-1}\right)$ 1636, 1600, 1552, and 1119. ${ }^{1} \mathrm{H}-\mathrm{NMR}\left(\mathrm{CDCl}_{3}, 400 \mathrm{MHz}\right), \delta_{\mathrm{H}} \mathrm{ppm}: 8.09(1 \mathrm{H}, \mathrm{s}, \mathrm{H}-$ 4), $7.68(1 \mathrm{H}, d, J=8.1 \mathrm{~Hz}, \mathrm{H}-10), 7.52(1 \mathrm{H}, t, J=7.8 \mathrm{~Hz}, \mathrm{H}-8), 7.36(1 \mathrm{H}, d, J=8.6 \mathrm{~Hz}, \mathrm{H}-7)$, $7,15(1 \mathrm{H}, t, J=7.8 \mathrm{~Hz}, \mathrm{H}-9), 6.56\left(1 \mathrm{H}, s, \mathrm{H}-3^{\prime}\right), 6.10\left(1 \mathrm{H}, \mathrm{s}, \mathrm{H}-6^{\prime}\right), 5.79$ and $5.75\left(2 \mathrm{H}, s_{,} 4^{\prime}-\right.$ $\left.\mathrm{O}-\mathrm{CH}_{2}-\mathrm{O}-5^{\prime}\right), 5.36\left(1 \mathrm{H}, \mathrm{s}, \mathrm{H}-7^{\prime}\right), 3.96\left(3 \mathrm{H}, \mathrm{s}, 2^{\prime}-\mathrm{OCH}_{3}\right), 3.81\left(3 \mathrm{H}, \mathrm{s}, 6-\mathrm{NCH}_{3}\right), 3.51(1 \mathrm{H}, d d$, $\left.J=9.7,4.4 \mathrm{~Hz}, \mathrm{H}-8^{\prime}\right), 3.38\left(1 \mathrm{H}, t, J=9.8 \mathrm{~Hz}, \mathrm{H}-9{ }^{\prime} \mathrm{b}\right), 3.25\left(1 \mathrm{H}, d d, J=10.1,4.4 \mathrm{~Hz}, \mathrm{H}-9^{\prime} \mathrm{a}\right)$, $3.16(1 \mathrm{H}, t, J=10.1 \mathrm{~Hz}, \mathrm{H}-9 \mathrm{~b}), 2.45(3 \mathrm{H}, s, \mathrm{H}-1) .{ }^{13} \mathrm{C}-\mathrm{NMR}\left(\mathrm{CDCl}_{3}, 100 \mathrm{MHz}\right), \delta_{\mathrm{C}} \mathrm{ppm}$ : 198.2 (C-2), 160.2 (C-5), 150.6 (C-2'), 147.1 (C-4'), 146.2 (C-10b), 140.9 (C-5'), 140.4 (C6a), 135.9 (C-3), 133.1 (C-4), 131.5 (C-8), 126.3 (C-10), 123.7 (C-4a), 123.0 (C-9), 120.5 (C$\left.1^{\prime}\right), 120.0$ (C-10a), $114.7(\mathrm{C}-7), 107.8\left(\mathrm{C}-6^{\prime}\right), 101.1\left(4^{\prime}-\mathrm{O}-\mathrm{CH}_{2}-\mathrm{O}-5^{\prime}\right), 95.0\left(\mathrm{C}-3^{\prime}\right), 72.5\left(\mathrm{C}-9^{\prime}\right)$, $58.2\left(9^{\prime}-\mathrm{OCH}_{3}\right), 56.7\left(2^{\prime}-\mathrm{OCH}_{3}\right), 37.3\left(\mathrm{C}-8^{\prime}\right), 33.0\left(\mathrm{C}-7^{\prime}\right), 30.0\left(6-\mathrm{NCH}_{3}\right), 25.8(\mathrm{C}-1)$. HRESIMS $\mathrm{m} / \mathrm{z} 448.1752[\mathrm{M}+\mathrm{H}]^{+}$calculated for $\mathrm{C}_{26} \mathrm{H}_{25} \mathrm{NO}_{6} \mathrm{~m} / \mathrm{z} 448.1760$.

\section{Conclusions}

In summary, three unreported quinolinone alkaloids: melicodenines $\mathrm{J}-\mathrm{L}$ (1-3), along with six known compounds (4-9), were isolated from Melicope denhamii leaves. The cytotoxicity activity of compounds (1-9) was evaluated against MCF-7, HeLa, and P-388 cells. Compounds $\mathbf{1}$ and $\mathbf{5}$ showed high activity against HeLa cells.

\section{Disclosure statement}

No potential conflict of interest was reported by the authors. 


\section{Funding}

This research financially supported by Universitas Airlangga, Surabaya, Indonesia (Hibah Mandat 2019 by Tjitjik Srie Tjahjandarie).

\section{References}

Chen J-J, Duh C-Y, Huang H-Y, Chen I-S. 2003. Furoquinoline alkaloids and cytotoxic constituents from the leaves of Melicope semecarpifolia. Planta Med. 69(6):542-546.

George S, Venkataraman R, Baby S. 2016. Melicodenine I, a new quinolinone alkaloid from Melicope denhamii leaves. Nat Prod Res. 31(8):890-895.

Holla H, Jenkins ID, Neve JE, Pouwer RH, Pham N, Teague SJ, Quinn RJ. 2012. Synthesis of melicodenines C, D and E. Tetrahedron Lett. 53(52):7101-7103.

Johns SR, Lamberton JA, Price JR, Sioumis AA. 1968. Identification of coumarins isolated from Lepiniopsis ternatensis (Apocynaceae), Pterocaulon sphacelatum (Compositae), and Melicope melanophloia (Rutaceae)*. Aust J Chem. 21(12):3079-3080.

Kamperdick C, Van NH, Sung TV, Adam G. 1999. Bisquinolinone alkaloids from Melicope ptelefolia. Phytochemistry. 50(1):177-181.

Nakashima K, Oyama M, Ito T, Akao Y, Witono JR, Darnaedi D, Tanaka T, Murata J, linuma M. 2012. Novel quinolinone alkaloids bearing a lignoid moiety and related constituents in the leaves of Melicope denhamii. Tetrahedron. 68(10):2421-2428.

Nakashima K, Oyama M, Ito T, Witono JR, Darnaedi D, Tanaka T, Murata J, linuma M. 2011. Melicodenines $A$ and $B$, novel Diels-Alder type adducts isolated from Melicope denhamii. Tetrahedron Lett. 52(36):4694-4696.

Rasamison VE, Brodie PJ, Merino EF, Cassera MB, Ratsimbason MA, Rakotonandrasana S, Rakotondrafara A, Rafidinarivo E, Kingston DGl, Rakotondraibe HL. 2016. Furoquinoline alkaloids and methoxyflavones from the stem bark of Melicope madagascariensis (Baker) T.G. Hartley. Nat Prod Bioprospect. 6(5):261-265.

Saputri RD, Tjahjandarie TS, Tanjung M. 2018. Meliglabrin, a new flavonol derivative from the leaves of Melicope glabra (Blume) T. Nat Prod Sci. 24(3):155-158.

Saputri RD, Tjahjandarie TS, Tanjung M. 2021. Two novel coumarins bearing an acetophenone derivative from the leaves of Melicope quercifolia. Nat Prod Res. 35(8):1256-1261.

Tanjung M, Rachmadiarti F, Saputri RD, Tjahjandarie TS. 2018. Mesucalophylloidin, a new isoprenylated 4-phenylcoumarin from Mesua calophylloides (Ridl.) Kosterm. Nat Prod Res. 32(9): 1062-1067.

Tanjung M, Tjahjandarie TS, Saputri RD, Kurnia BD, Rachman MF, Syah YM. 2021. Calotetrapterins A-C, three new pyranoxanthones and their cytotoxicity from the stem bark of Calophyllum tetrapterum Miq. Nat Prod Res. 35(3):407-412.

Tjahjandarie TS, Tanjung M, Saputri RD, Aldin MF, Susanti RA, Pertiwi NP, Wibawa RS, Halizah IN. 2021. Cytotoxicity evaluation of two new chalcones from the leaves of Flemingia macrophylla (Willd.) Merr. Phytochem Lett. 44:78-81.

Tjahjandarie TS, Tanjung M, Saputri RD, Rahayu DO, Gunawan ANI, Aldin MF. 2020. Two new 2arylbenzofurans from Sesbania grandiflora L. Nat. Prod. Res. :1-6. 